\title{
OPERATORS COMMUTING WITH POSITIVE OPERATORS
}

\author{
MEHDI RADJABALIPOUR
}

ABSTRACT. Necessary and sufficient conditions are obtained for an operator to commute with a positive operator.

Throughout the paper, by an operator we mean a bounded linear transformation acting in a Hilbert space $H$. The algebra of all operators in $H$ is denoted by $B(H)$.

Arveson's theorem [1] about transitive algebras states that if $\mathfrak{A}$ is a strongly closed transitive algebra of operators and if $\mathfrak{U}$ contains a maximal abelian selfadjoint algebra (with respect to $B(H)$ ), then $\mathfrak{A}=B(H)$. (A transitive algebra is one whose only invariant subspaces are $\{0\}$ and $H$.) Foiaş [2] gives a different proof of Arveson's theorem mainly based on the following facts:

(F1) If $\mathfrak{A}$ is a strongly closed proper subalgebra of $B(H)$, then $\mathfrak{A}$ leaves the range of a nonzero, noninvertible positive operator $K$ invariant. In particular, if $\mathfrak{A}$ contains a maximal abelian selfadjoint algebra $\mathfrak{R}$, then $K$ can be chosen such that $K \in \mathfrak{R}$ and $\mathfrak{U} K \subset K \mathfrak{A}$.

(F2) If $\mathfrak{A}$ is a uniformly closed algebra and $\mathfrak{A} K \subset K \mathfrak{A}$ for some noninvertible positive operator $K \neq 0$, then $\mathfrak{A}$ is not transitive.

In the proof of (F2) it is shown that if $E$ is the resolution of the identity for $K$ and $T \in \mathfrak{A}$, then $T E([t, \infty)) H \subset E([t / a, \infty)) H$ for $0<t<\|K\|$, where $a$ is a fixed number not less than 1. (For a similar result about decomposable operators see [3].) In the present paper, given a positive operator $K$, we study conditions on an arbitrary $T$ (not necessarily in an algebra) to satisfy the above condition, and also prove a kind of converse to our result. In fact, Theorem 1 shows that if $T$ is an operator and $a \geqslant 1$, and if

$$
\liminf _{n>0}\left(\left\|K^{-n} T K^{n}\right\| / a^{n}\right)<\infty
$$

then

$$
T E([t, \infty)) H \subset E([t / a, \infty)) H, \quad 0<t<\|K\| .
$$

Note that we assume $K^{-n} T K^{n}$ can be extended boundedly to all of $H$. In (1) and (2), $K$ is an injective positive operator and $E$ is its resolution of the identity. Conversely, Theorems 2 and 3 show that if $T$ satisfies (2), then (1) holds but for $a$ replaced by $a^{2}$. As corollaries, we obtain necessary and

Received by the editors May 15, 1978 and, in revised form, July 12, 1978.

AMS (MOS) subject classifications (1970). Primary 47B15, 47C05.

Key words and phrases. Hilbert space, positive operator, spectral projection, operator algebra. (c) 1979 American Mathematical Society 
sufficient conditions for an operator to commute with an injective positive operator.

THEOREM 1. Let $K$ be an injective positive operator and let $a \geqslant 1$. Then the following assertions are true.

(a) For every operator $T$, condition (1) implies condition (2).

(b) If condition (2) holds for all operators $T$ in some algebra $\mathfrak{A}$, then for each $t \in(0,\|K\|)$ the closure of $\mathfrak{A} E([t, \infty)) H$ is an invariant subspace of $\mathfrak{A}$, which is nontrivial if $0 \in \sigma(K)$ and $I \in \mathfrak{A}$.

(c) (Foiaş) If $\mathfrak{A} K \subset K \mathfrak{A}$ for some uniformly closed algebra $\mathfrak{A}$, then for each $t \in(0,\|K\|)$ the closure of $\mathfrak{A} E([t, \infty)) H$ is an invariant subspace of $\mathfrak{A}$ and $K$, which is nontrivial if $0 \in \sigma(K)$ and $I \in \mathfrak{A}$.

Proof. Assume without loss of generality that $\|K\|=1$ and $0<t<1$.

(a) Let $b<1 / a$ and let $K=K_{1} \oplus K_{2} \oplus K_{3}$ with respect to some orthogonal direct sum $H=H_{1} \oplus H_{2} \oplus H_{3}$ such that $\sigma\left(K_{1}\right) \subset[t, 1], \sigma\left(K_{2}\right) \subset[t b, t]$ and $\sigma\left(K_{3}\right) \subset[0, t b]$. Note that some of these spaces may be trivial. If $H_{3} \neq$ $\{0\}$, let $T_{31}=P_{3} T \mid H_{1}$, where $P_{3}: H \rightarrow H_{3}$ is the projection onto $H_{3}$. It follows that

$$
\begin{aligned}
\left\|T_{31}\right\| & =\left\|K_{3}^{n} K_{3}^{-n} T_{31} K_{1}^{n} K_{1}^{-n}\right\| \\
& \leqslant\left\|K_{3}^{n}\right\| \cdot\left\|K_{1}^{-n}\right\| \cdot\left\|K_{3}^{-n} T_{31} K_{1}^{n}\right\| \leqslant\left\|K^{-n} T K^{n}\right\| b^{n} .
\end{aligned}
$$

Thus $\left\|T_{31}\right\| \leqslant \lim \inf _{n>0}\left\|K^{-n} T K^{n}\right\| b^{n}=0$, and hence $T H_{1} \subset H_{1} \oplus H_{2} \subset$ $E([t b, 1]) H$. Therefore $T E([t, 1]) H \subset E([t b, 1]) H$ for all $b<1 / a$ and thus (2) follows.

(b) assume without loss of generality that $\mathfrak{A}$ contains the identity. Then for $t \in(0,1)$ the closure of $\mathfrak{A} E([t, 1]) H$ is a nonzero invariant subspace of $\mathfrak{A}$ (included in $E([t / a, 1]) H$ ).

(c) Here, again, assume without loss of generality that $\mathfrak{A}$ contains the identity. Since $\mathfrak{A}$ is uniformly closed and $K^{-1} T K \in \mathfrak{A}$ for all $T \in \mathfrak{A}$, it follows from the closed graph theorem that the map $W(T)=K^{-1} T K$ is a bounded operator in $\mathfrak{A}$ and hence

$$
\underset{n>0}{\lim \sup }\left\|K^{-n} T K^{n}\right\| /\|W\|^{n}<\infty \quad \text { for all } T \in \mathfrak{A} \text {. }
$$

For a fixed $t \in(0,1)$ let $M=\mathfrak{A} E([t, 1]) H$. In view of (b), the closure of $M$ is an invariant subspace of $\mathfrak{A}$. Let $x \in E([t, 1]) H$ and let $T \in \mathfrak{A}$. Let $K_{1}=$ $K \mid E([t, 1]) H$ and $K_{2}=K \mid E([t a, t)) H$. Since $K_{1}^{-1} x \in E([t, 1]) H$, it follows that $\left(K_{1} \oplus K_{2}\right)^{-1} T x=\left(K_{1} \oplus K_{2}\right)^{-1} T K K_{1}^{-1} x=K^{-1} T K K_{1}^{-1} x \in M$ and thus $\left(K_{1} \oplus K_{2}\right)^{-1} M \subset M$. Hence the closure of $M$ is an invariant subspace of $\left(K_{1} \oplus K_{2}\right)^{-1}$ and thus of $K_{1} \oplus K_{2}$, because $K_{1} \oplus K_{2}$ is Hermitian.

COROLlaRY 1. Let $K$ be a nonscalar, injective positive operator and assume $\lim \inf _{n>0}\left\|K^{-n} T K^{n}\right\|<\infty$ for some operator $T$. Then $T$ has a nontrivial invariant subspace. 
THEOREM 2. Let $K$ be an injective positive operator with the resolution of the identity E. Assume (2) holds for some operator $T$ and some $a>1$. Then

$$
\left\|K^{-n} T K^{n}\right\|<\left(a^{3 n} /\left(a^{n}-1\right)\right)\|T\| \quad(n=1,2,3, \ldots) .
$$

Proof. Assume without loss of generality that $\|K\|=1$. Let $b=1 / a$. Let $H_{1}=E([b, 1]) H$ and $H_{i}=E\left(\left[b^{i}, b^{i-1}\right)\right) H$ for $i=2,3, \ldots$ Note that some $H_{i}$ may be trivial. Let $J=\left\{i: H_{i} \neq\{0\}\right\}$. For $i, j \in J$, let $K_{i}=K \mid H_{i}$ and $T_{i j}=P_{i} T \mid H_{j}$, where $P_{j}: H \rightarrow H_{j}$ is the projection onto $H_{j}$. By the hypotheses, $T_{i j}=0$ for $i \geqslant j+2$. For $i \leqslant j+1$ and $i, j \in J$ we have

$$
\begin{gathered}
K_{i}^{-n} T_{i j} K_{j}^{n}=P_{j} K^{-n} T K^{n} \mid H_{i}, \\
\left\|K_{i}^{-n} T_{i j} K_{j}^{n}\right\| \leqslant b^{-n i} b^{n j-n}\|T\|=b^{n(j-i-1)}\|T\|,
\end{gathered}
$$

for $n=1,2, \ldots$ Let $C=\left(\left(c_{i j}\right)\right)$ be the matrix in which

$$
\begin{aligned}
& c_{i j}=0 \quad \text { if } i \geqslant j+2, \\
& c_{i j}=\|T\| b^{n(j-i-1)} \quad \text { if } i<j+2 .
\end{aligned}
$$

Obviously

$$
\|T\|^{-1} C=b^{-2 n} S+b^{-n} I+\sum_{0<k} b^{n k}\left(S^{*}\right)^{k+1},
$$

where $S$ is a unilateral shift. Hence $\|C\| \leqslant\|T\| b^{-2 n} /\left(1-b^{n}\right)$. Since $K^{-n} T K^{n}=\left(\left(K_{i}^{-n} T_{i j} K_{j}^{n}\right)\right)_{i, j \in J}$ is majorized by the compression $\left(\left(c_{i j}\right)\right)_{i, j \in J}$ of $\left(\left(c_{i j}\right)\right)$, it follows from [3, Lemma 1$]$ that

$$
\left\|K^{-n} T K^{n}\right\| \leqslant \frac{b^{-2 n}}{1-b^{n}}\|T\|=\frac{a^{3 n}}{a^{n}-1}\|T\|
$$

for $n=1,2, \ldots$.

The interesting case of $a=1$ is treated in the following theorem and corollaries.

THEOREM 3. Let $K$ be an injective positive operator with the resolution of the identity $E$ and let $T$ be an arbitrary operator. Assume $T E([t, \infty))=$ $E([t, \infty)) T E([t, \infty))$ for all $t>0$. Then $\left\|K^{-n} T K^{n}\right\|<4\|T\|$ for $n=$ $1,2, \ldots$

Proof. Assume without loss of generality that $\|K\|=1$. Let $b=2^{-1 / n}$ and let $H_{i}, K_{i}$, and $T_{i j}$ be as in the proof of Theorem 2 . Here $T_{i j}=0$ for $i>j+1$. Following the proof of Theorem 2, we obtain

$$
\left\|K^{-n} T K^{n}\right\| \leqslant\left(b^{-n} /\left(1-b^{n}\right)\right)\|T\|=4\|T\|, \quad n=1,2, \ldots
$$

COROLlary 2. Let $K$ be an injective positive operator with the resolution of the identity $E$, and let $T$ be an arbitrary operator. Then $T E([t, \infty))=$ $E([t, \infty)) T E([t, \infty))$ for all $t>0$ if and only if $\left\|K^{-n} T K^{n}\right\|$ is uniformly bounded for $n=1,2, \ldots$. 
COROllary 3. Let $K$ be an injective positive operator and let $T$ be an arbitrary operator. Then $T K=K T$ if and only if $\left\|K^{-n} T K^{n}\right\|$ is uniformly bounded for $n= \pm 1, \pm 2, \pm 3, \ldots$.

The last corollary has the following generalization.

Corollary 4. Let $K$ and $L$ be two injective positive operators and let $T$ be an arbitrary operator. Then $K T=T L$ if and only if $\left\|K^{-n} T L^{n}\right\|$ is uniformly bounded for $n= \pm 1, \pm 2, \pm 3, \ldots$.

Proof. Consider the operators

$$
\left(\begin{array}{ll}
K & 0 \\
0 & L
\end{array}\right) \text { and }\left(\begin{array}{cc}
0 & T \\
T^{*} & 0
\end{array}\right)
$$

and apply Corollary 3.

Added in Proof (SePtember 1978). A special case of Theorem 3 in which $K$ is boundedly invertible is proved by James A. Deddens in a paper entitled Another description of nest algebras to appear in Proc. Long Beach Conf., June 1977, Springer, New York. Our proof given here is much shorter. Using Deddens' result, J. P. Williams obtains our Corollary 3 for invertible $K$.

\section{REFERENCES}

1. W. B. Arveson, $A$ density theorem for operator algebras, Duke Math. J. 23 (1967), 635-648.

2. C. Foias, Invariant para-closed subspaces, Indiana Univ. Math. J. 21 (1972), 887-906.

3. A. A. Jafarian, Algebras intertwining normal and decomposable operators, Canad. J. Math. (to appear).

4. E. Nordgren, M. Radjabalipour, H. Radjavi and P. Rosenthal, On invariant operator ranges, Trans. Amer. Math. Soc. 251 (1979), 389-398.

Department of Mathematics, University of Northern Iran, P. O. Box 444, Babolsar, IRAN 